\title{
Rain Granularity Effects on Bandwidth Demand for Faded DVB-RCS Systems
}

\author{
Mohamed Al-Mosawi*, ${ }^{*}$, Rinat Khusainov, Boris Gremont \\ School of Engineering, University of Portsmouth, Portsmouth, UK.
}

\begin{abstract}
SUMMARY
Broadband satellite communication networks, operating at $\mathrm{Ka}$ band and above, play a vital role in today's worldwide telecommunication infrastructure. The problem, however, is that rain can be the most dominant impairment factor for radio propagation above $10 \mathrm{GHz}$. This paper, studies bandwidth and timeslot allocation problem for rain faded DVB-RCS satellite networks. We investigate how using finer rain granularity can improve bandwidth utilization in DVB-RCS return links. The paper presents a mathematical model to calculate the bandwidth on demand. We formulate the radio resource allocation as an optimization problem and propose a novel algorithm for dynamic carrier bandwidth and time slots allocation, which works with CBR type of traffic. We provide theoretical analysis for the time slot allocation problem and show that the proposed algorithm achieves optimal results. The algorithm is evaluated using a MATLAB simulation with historical rain data for the UK. Copyright (C) 2013 John Wiley \& Sons, Ltd.
\end{abstract}

Received ...

KEY WORDS: DVB-RCS; fade mitigation techniques; MF-TDMA; time-slot allocation; dynamic radio resource allocation; optimization problems.

\section{INTRODUCTION}

VSAT networks provide high-speed multimedia services, including voice, video and Internet for a vast number of subscribers distributed over a very wide area. The DVB-RCS stands for Digital Video Broadcast Return Channel via Satellite [1,2], which is a centralized communication system. There is a single station called Network Control Centre (NCC) that controls all communication processes in real time.

European DVB-RCS systems providers use the following access mechanisms recommended by the European Telecommunications Standards Institute (ETSI) [1, 2].Time Division Multiple Access (TDMA) is used on the forward link as the access mechanism, while the return link is shared by earth stations using an Multi-Frequency Time Division Multiple Access (MF-TDMA) scheme. Typically, Ku band (12-18 GHz) is used for the forward link and Ka band (18-30 GHz) for the return link $[1,2,3]$.Forward link transmissions for user terminals are organized in bursts. The bursts are assumed to be made of a fixed number of time-slots, which are long enough to transmit one fixed-size packet.

Satellite links are affected severely by rain fading, which reduces the link capacity. NCC has to make sure that extra time slots or extra bandwidth are available to provide users with the requested quality of service in varying weather conditions. Consequently, efficient Radio Resource Management (RRM) and bandwidth utilization under rainy conditions have become important research topics in satellite telecommunications.

\footnotetext{
*Correspondence to: Mohamed Al-Mosawi, School of Engineering, University of Portsmouth, Portsmouth, UK.

$\dagger$ mohamed.al-mosawi@port.ac.uk 
Combinatorial optimization has been used to address the problem of efficient resource allocation in DVB-RCS satellite systems. In [4], resource allocation for rain faded forward links has been modeled as a knapsack problem. We look into resource allocation for DVB-RCS return links. The difference is that here we work with MF-TDMA frames instead of TDMA.

The main objective of this paper is to investigate how using finer rain granularity can improve bandwidth utilization in DVB-RCS return links. Our contributions are as follows:

- We study the effects of using finer rain granularity on the total bandwidth demand in the case of constant data rate traffic and demonstrate the resulting improvements on real historical rain data.

- We propose a novel low-cost time slot allocation algorithm for bursty data rate traffic to achieve a better bandwidth utilization using finer rain granularity, while still meeting the Quality of Service $(\mathrm{QoS})$ requirements.

- We provide theoretical analysis of the time slot allocation problem and show that the proposed algorithm achieves theoretically optimal assignment.

- We evaluate the proposed algorithm using a simulation with real historical rain data to demonstrate its practical benefits.

The current version of the algorithm deals with the guaranteed throughput service. The guaranteed throughput service is defined as the service which ensures that a subscriber always gets the bandwidth requested regardless of traffic behavior of other users [3].

\section{VSAT SYSTEMS}

\subsection{Network architecture}

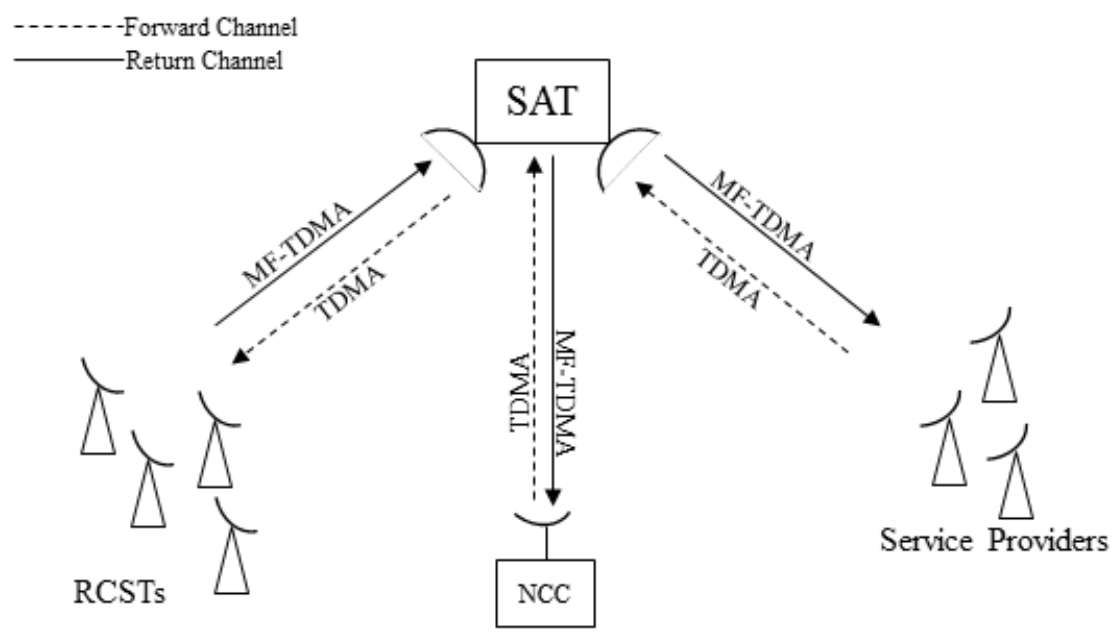

Figure 1. Network architecture

The network under consideration is depicted in Figure 1. The satellite network mesh consists of the following elements:

1. A Geo satellite that operates at Ka-band for earth space communications.

2. Users, which are called Return Channel via Satellite Terminals (RCST).

3. Network Control Centre (NCC), a single station that orchestrates the interactions between RCSTs and service providers, and is responsible for resource allocation for active RCSTs. 


\subsection{Return channel description}

Multi-Frequency Time Division Multiple Access (MF-TDMA) is the media access scheme used in DVB-RCS systems. MF-TDMA is a hybrid system, in which RCSTs share time as well as frequency. The scheme combines Frequency Division Multiple Access (FDMA) with Time Division Multiple Access (TDMA), which allows better utilization of the limited bandwidth available. MF-TDMA scheme divides bandwidth into a number of carriers, and each carrier is divided into time slots.

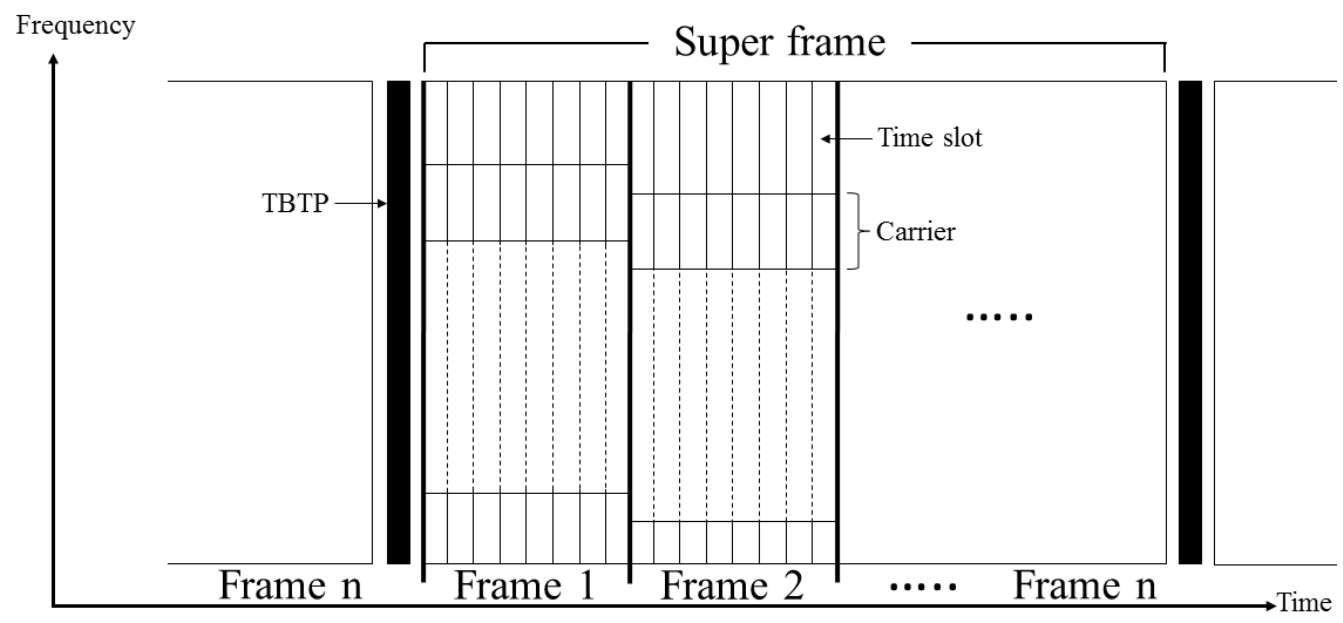

Figure 2. Simplified dynamic structure of MF-TDMA

The MF-TDMA structure in DVB-RCS systems is organized into three levels: Super Frame (SP), Frame, and Time-slots, as shown in Figure 2. A super frame consists of a number of frames, and identified by a unique Superframe_ID. Similarly, each frame consists of a number of time slots and has a unique frame_ID. A time slot is defined by centre frequency, bandwidth, and duration.

There are two MF-TDMA frame structures described in literature: fixed, and dynamic. The fixed structure is sub-divided into constant frequency bandwidth described in [5, 6], and constant rate capacity used in[7]. Both are with a fixed number of time-slots.

In this paper we propose a dynamic frame structure. In our framework, we impose the following constraints.

1. All super frames have the same number of frames.

2. All frames have the same bandwidth, and time duration.

3. All carriers within a frame have the same number of time slots.

4. All time slots have the same guaranteed capacity [bits/second].

5. The number of carriers in each frame varies depending on the bandwidth requests calculated by the NCC. That leads to a variable number of time slots per frame. Having a different number of time slots per frames means a different capacity for each frame.

In the communication scenario, the NCC sends Terminal Burst Time Plan (TBTP) to all RCSTs at the beginning of each super frame to update transmission parameters. TBTP for each RCST includes frame_ID, transmission center frequency, bandwidth, time, and duration that RCST will use to transmit its packets.

\section{RAIN FADE MITIGATION AT PHYSICAL LAYER}

Rain is a dominant factor in radio propagation above $10 \mathrm{GHz}$. Different Fade Mitigation Techniques (FMT)s are available to compensate rain fading. In [5, 8] the authors use Adaptive Coding and Modulation (ACM), which adjusts transmitted signal and the required bandwidth dynamically according to link's attenuation level. In [6], the Forward Error Correction (FEC) was implemented, 
which allocates more time to rain faded links to transmit their data while keeping the allocated bandwidth constant, accompanied with changes in transmission parameters. There are two main trends in today's rain fading research. first approach is to consider a variable total link capacity due to rain fading. The second approach studies the rain effects on the RCST links individually.

Transmission parameters updates are based on Signal-to-Noise Ratio (SNR) estimation. Authors in [6] propose a model to predict link SNR based on a statistical spatial and temporal analysis of rain fading. In [6], the SNR is estimated using Markov-Model, based on the worse case link condition, which may lead to a waste in bandwidth. Other researchers estimate SNR by averaging the SNR values measured for a given duration [9].

In this paper, Adaptive Coding and Modulation for satellite links (ACM) is employed based on the instantaneous level of rain fading measured in each individual RCST link. The transmission parameters are updated prior to each single superframe, in other words, each 0.5 second (Superframe duration). In ACM calculation process, the rain fade obtrained is round up to the most significant value in order to compensate any degradation in link quality while transmitting data. On the other hand, in case of no available weather data the algorithm by design can adapt to any available prediction or interpolation methods used to calculate rain fading.

ACM is a resource-shared mitigation technique. It modifies signals transmitted by earth stations in the case of link quality degradation. ACM always tries to maintain an agreed level of bit-error ratio (BER), while allowing the reduction of required energy per bit when the link suffers fading conditions.

One of the coding methods that attracted the satellite communication industry is the parallel concatenated coding with interleaving (i.e. turbo coding), which led eventually to the standardization of these methods of coding in the second generation DVB-RCS earth stations [5]. An advantage of such coding is the extension of the dynamic range of the FMT, which leads to an increase in connection's throughput during the unfaded link condition,[5]. However, when the carrier-to-noise power spectral density decreases, lower coding rate and/or reduced constellation will be selected due to the propagation effects.

The ACM can be classified into two types: constant user bit rate, that is the scenario of nonnegotiable bit rate (i.e. voice or video transmission); and constant bandwidth, when the allocated bandwidth for each connection cannot be changed. ACM can provide a dynamic range of 0 to 17 $\mathrm{dB}$ depending on the link power budget, described in[5].

Depending on link's attenuation level, the related ACM mode is chosen from a range of available ACM schemes as in Table 1. The system always tries to keep the BER below $10^{-7}$ therefore a more robust ACM scheme is selected when the CNR degrades.

As mentioned before the ACM can cover a dynamic range of $17 \mathrm{~dB}$. Any change in ACM must be accompanied by a change in bandwidth as shown in Table 1 . Moreover, only DVB-RCS2 can cover the $17 \mathrm{~dB}$ dynamic range of ACM, while the original DVB-RCS can only work in the range of 4 to 14 from Table 1, so in the case of using the power limited systems, the DVB-RCS therefore could be used, which means reducing the link power budget required by $9 \mathrm{~dB}$ and the dynamic range of the FMT will fall to $8 \mathrm{~dB}$.

\section{RAIN GRANULARITY FOR CBR TRAFFIC}

When studying rain fading, researchers use mathematical models to describe the problem. Resource allocation problem is presented as linear programming equation in [6, 8, 10]. In [10], more complicated data traffic was considered. The data traffic had different delay classes, which gave them different packing priority levels. The authors suggested a heuristic algorithm to solve the allocation problem. However, RCSTs were divided into only two groups: rainy and non-rainy terminals; presuming the worst case conditions for the RCSTs affected by rain. This approach leads to a waste in bandwidth due to overestimation of bandwidth requirements. Authors in [6] suggest solving the allocation problem using bin-packing algorithms. In [7] the RCSTs are allocated the maximum bandwidth at any given time leading to a Constant Bandwidth Allocation (CBA). This approach is also not spectral efficient. We propose to distinguish between more than two levels or 


\begin{tabular}{|c|c|c|c|c|}
\hline Mode $\mathrm{m}$ & Modulation & $\rho$ & $B$ & Rain protection $(A t t)[\mathrm{dB}]$ \\
\hline$\overline{1}$ & \multirow{4}{*}{ 16APSK } & $9 / 10$ & $\overline{\overline{B^{c}}}$ & 1.3 \\
\hline 2 & & $5 / 6$ & $1.08 B^{c}$ & 2.7 \\
\hline 3 & & $3 / 4$ & $1.2 B^{c}$ & 4.2 \\
\hline 4 & & $2 / 3$ & $1.35 B^{c}$ & 5.7 \\
\hline 5 & \multirow{3}{*}{ 8PSK } & $4 / 5$ & $1.5 B^{c}$ & 6.2 \\
\hline 6 & & $3 / 4$ & $1.6 B^{c}$ & 7 \\
\hline 7 & & $2 / 3$ & $1.8 B^{c}$ & 8.5 \\
\hline 8 & \multirow{8}{*}{ QPSK } & $6 / 7$ & $2.1 B^{c}$ & 9.6 \\
\hline 9 & & $4 / 5$ & $2.25 B^{c}$ & 10.5 \\
\hline 10 & & $3 / 4$ & $2.4 B^{c}$ & 11.3 \\
\hline 11 & & $2 / 3$ & $2.7 B^{c}$ & 12.4 \\
\hline 12 & & $3 / 5$ & $3 B^{c}$ & 13.2 \\
\hline 13 & & $1 / 2$ & $3.6 B^{c}$ & 14.4 \\
\hline 14 & & $2 / 5$ & $4.5 B^{c}$ & 15.9 \\
\hline 15 & & $1 / 3$ & $5.4 B^{c}$ & 17 \\
\hline 16 & BPSK & $1 / 2$ & $7.2 B^{c}$ & 17.6 \\
\hline
\end{tabular}

Table I. DVB-RCS2 supported rate variation depending on selected ACM mode [5]

rain and utilize this for bandwidth allocation. More specifically, instead of using only two groups, rainy and non-rainy, we assign RCSTs to the available rain levels supported by the ACM fade mitigation technique. In this Section, we study how using this finer rain granularity can affect the total bandwidth demand for the Constant Bit Rate traffic.

\subsection{Mathematical Formulation}

When considering rain granularity, there is no clear indication of how the upper bound of the required bandwidth is determined. This can be measured experimentally using historical data for a given RCSTs set.

The mathematical model is based on the assumption of Constant Bit Rate data traffic (CBR) i.e., RCSTs transmit at peak rate at all times. In other words, all time slots per carrier are reserved for one RCST. In the present model, ACM is used as an FMT. Table 1 states the modulation parameters for each operation mode. Using worst case scenario for rain fading means for a given data rate request $R_{i}$ by RCST $i$, the allocated bandwidth is $B^{c}$ in clear sky condition, and $B^{r}$ in the case of rain, $B^{r}=7.2 B^{c}$, from Table 1 . Hence the bandwidth $B_{i}^{c}$ required to satisfy the data rate requet $R_{i}$ in clear sky condition, is calculated using Equation 2 derived from [5]:

$$
B_{i}^{c}=\frac{R_{i} \cdot(1+\alpha)}{\rho\left(A t t_{i}\right) \cdot \log _{2} M\left(A t t_{i}\right)},[H z]
$$

Where $\rho, M$ are transmission parameters incorporated with clear sky condition, and $\alpha$ is the roll of factor, $\alpha \simeq 0.35$.

Let's denote $x_{i, j}$, a scalar indicating whether user $i$ has $j$ rain fading level or not. Hence, the experimental upper bound for the total bandwidth on demand is the summation of all user's bandwidth requests, $B o D$ is calculated is as follows.

$$
\begin{gathered}
B o D=\sum_{i=1}^{u}\left(x_{i, 1} \cdot B_{i}^{c}+\left(x_{i, 1}-1\right) \cdot B_{i}^{r}\right),[H z] \\
x_{i, j} \in\left\{\begin{array}{l}
1, \text { if RCST } i \in \text { rainleve } j . \\
0, \text { otherwise. }
\end{array}\right.
\end{gathered}
$$

where $u$ is the total number of RCSTs. $x_{i, 1}=1$ indicates that RCST $i$ has clear sky condition.

Considering finer rain fading granularity, at any given time, there will be a number of RCSTs suffering $k$ or less levels of rain fading. For each fading level $j, 1 \leq j \leq k$, the NCC allocates 
different amount of bandwidth per carrier for each RCST, and sets transmission parameters. $B_{i}^{j}$ is the bandwidth allocated to RCST $i$ to mitigate $j$ rain fading level. Total amount of bandwidth on demand $B o D^{g}$ considering rain granularity is as follows:

$$
B o D^{g}=\sum_{i=1}^{u}\left(\sum_{j=1}^{k} x_{i, j} \cdot B_{i}^{j}\right),[H z]
$$

In bandwidth allocation process, RCST $i$ is assumed to have one rain fading level at a given time instant. This constraint is shown as follows:

$$
\begin{gathered}
\text { s.t } \sum_{j=1}^{k} x_{i, j}=1, \forall i \\
\sum_{i=1}^{u}\left(\sum_{j=1}^{k} x_{i, j} \cdot B_{i}^{j}\right) \leq c,
\end{gathered}
$$

where $c$ is the total bandwidth available in Hertz.

The constraint in Equation 7 means that the total amount of used bandwidth by requests should not exceed the available bandwidth.

\subsection{Simulation Setup}

In the simulation, the city-oriented RCSTs geographical distribution suggested in [8] is used, where 120 RCSTs are used with geographical distribution pattern of: $75 \%$ of the RCSTs are located in 4 different cities: London, Manchester, Dublin, Glasgow, and the rest is randomly distributed all over the UK with the minimum separation distance of $1 \mathrm{~km}$ between RCSTs, illustrated in Figure 3.

RCSTs set up connections with data rate of $144 \mathrm{~Kb} / \mathrm{s}$ (topology A) guaranteed throughput of a low rate video streaming traffic type. The operation frequency of the system is $30 \mathrm{GHz}$, which is the highest frequency used in Ka-band [6]; hence, maximum rain attenuation is expected.

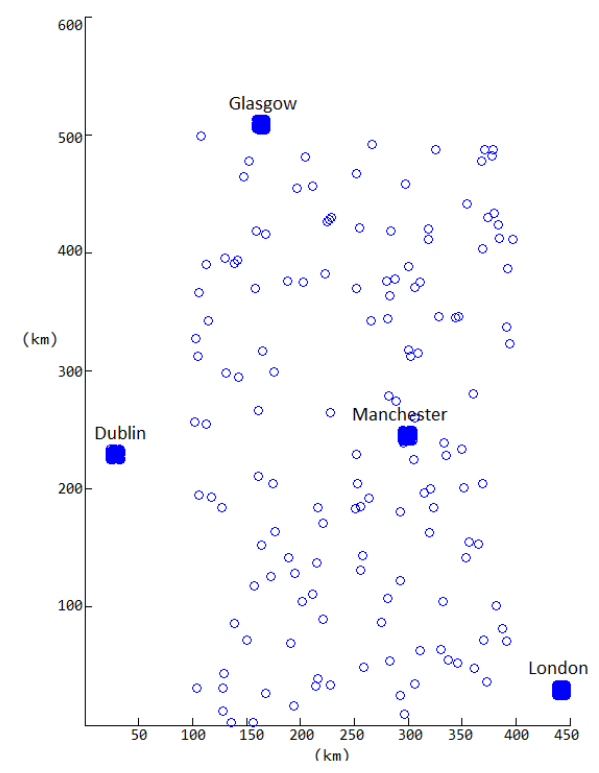

Figure 3. Users geographical distribution 


\subsection{Numerical results}

Figure 4 shows the amount of used bandwidth for the given distribution of RCSTs for worse case scenario in (a), and considering finer rain granularity in (b). The bottom level of the used bandwidth corresponds to the minimum rain fading level (i.e. no rain) is the same in both cases. The overall bandwidth usage statistics can be represented as a Cumulative Density Function (CDF) of the total bandwidth used. This is shown in Figure 5.
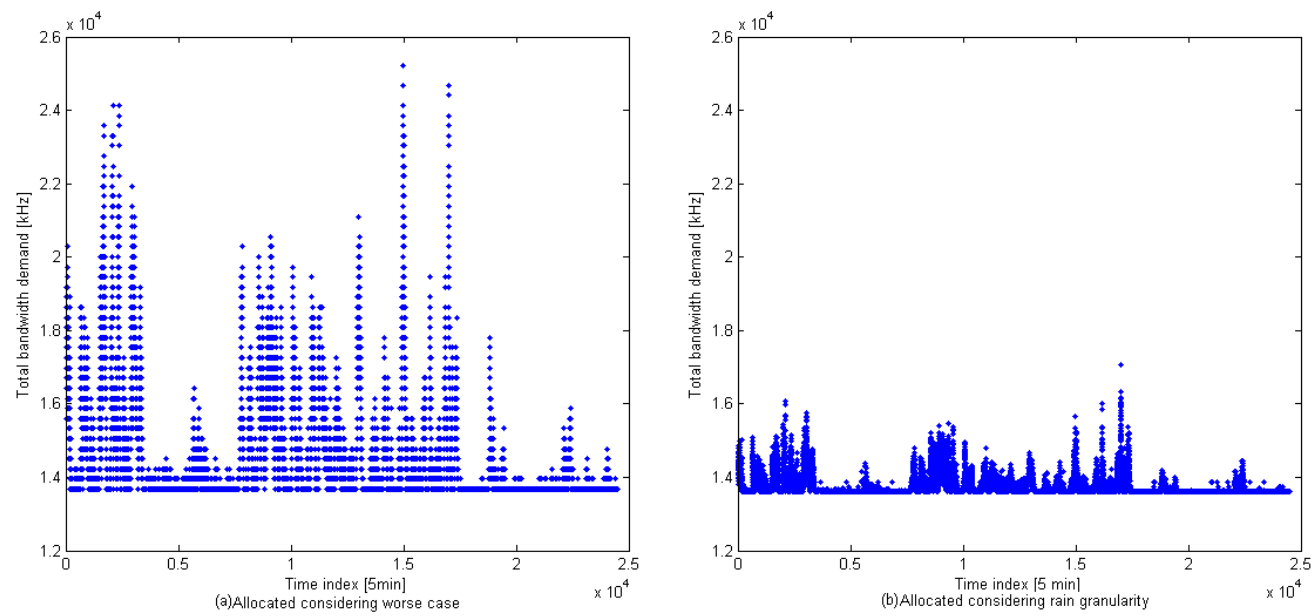

Figure 4. Total bandwidth demand
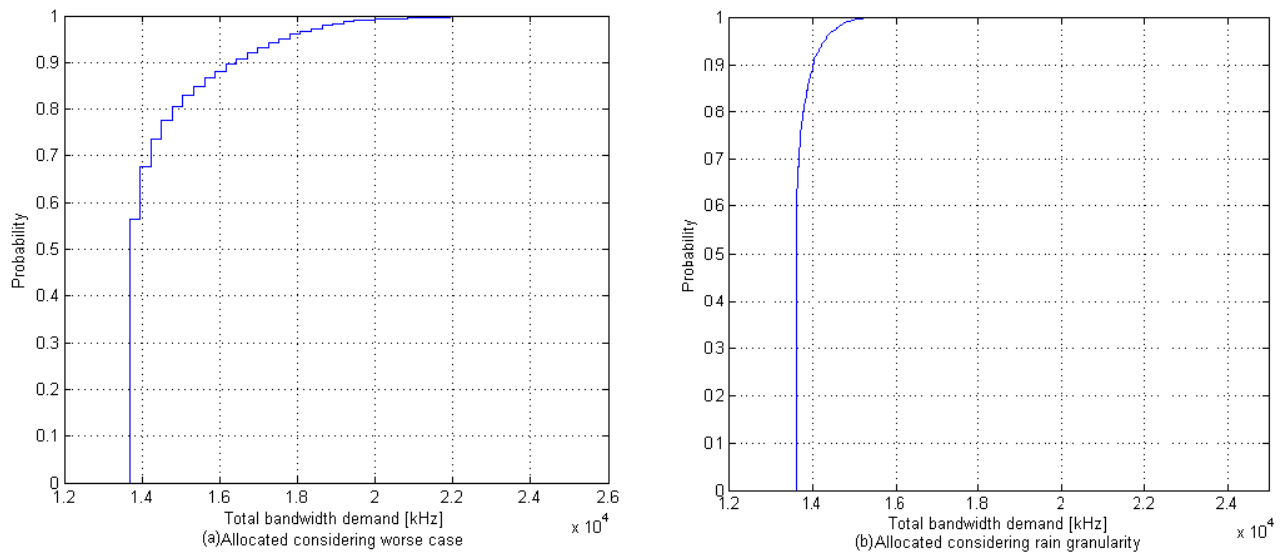

Figure 5. Total bandwidth demand CDF

As it can be seen from the results shown in Figure 4 and 5, at any given time,the total bandwidth required for the worst case scenario is up to $25.23 \mathrm{MHz}$, whereas in the case of using finer rain granularity the total required bandwidth is below $17.07 \mathrm{MHz}$. Therefore, using finer rain granularity in the given scenario improves bandwidth utilization by $33 \%$.

In the case of using Constant Bandwidth Allocation (CBA) the reserved bandwidth for the given scenario is $46.66 \mathrm{MHz}$ calculated using Equation 2 and Table 1. Thus, using finer rain granularity would achieve $64 \%$ improvement in bandwidth utilization compared to CBA. 


\section{RAIN GRANULARITY FOR BURSTY TRAFFIC}

The results given in Section 4 demonstrate the effects of taking into account rain conditions on the total bandwidth demand. Although it shows the effect of rain granularity, but it does not reflect a real traffic situation since RCSTs can be active, idle, or offline. Burstiness, peak rate, and average rate are parameters describing users traffic. Authors in [3] describe these models. Users requests are statistically multiplexed and transmitted together in real time, they share the same available bandwidth to achieve utilization. Fluid flow, Gaussian are approaches used to estimate the bandwidth usage, calculating what authors in [3,11, 12, 13] called equivalent bandwidth, which stands for the estimated total data bit rate requested by all RCSTs. Then the total frequency bandwidth required to satisfy the calculated equivalent data bit rate is allocated based on the worst rain fading scenario.

In this paper, we approach the statistical multiplexing from a different angle. We measure the requested data rate in real time and use the actual rain fall rate to determine the maximum required frequency bandwidth for the requested data rate.

\subsection{Mathematical Formulation}

The authors in [8] present the problem of resource allocation with rain fading as a combinatorial optimization problem, aiming to pack optimally packets of different sizes into the MF-TDMA frame space. In this paper, we propose a dynamic frame structure explained in Section 2.2. Table 2 explains the meaning of different symbols used in the subsequent equations.

\begin{tabular}{|c|l|}
\hline Symbol & Definition \\
\hline \hline$u$ & The total number of RCSTs supported by the network. \\
\hline$k$ & The number of available ACM modes in the network. \\
\hline$r_{i}$ & The rain fading level for RCST $i$. \\
\hline$p_{i}$ & Number of packets buffered by RCST $i$. \\
\hline$c$ & The available MF-TDMA bandwidth in Hertz. \\
\hline$f\left(r_{i}\right)$ & The bandwidth assigned by BoD to mitigate level $r_{i}$ rain fading. \\
\hline$L$ & The number of available MF-TDMA frames per super frame. \\
\hline$T$ & The duration of an MF-TDMA time-slot. \\
\hline$R_{i}$ & Requested data rate by RCST $i$. \\
\hline$w_{i, s}$ & The bandwidth required for a time slot $s$ in carrier $i$. \\
\hline $\mathrm{S}$ & The total number of time slots in a super frame (calculated as L $*$ time slots per frame) \\
\hline $\mathrm{N}$ & Number of carrier for a given frame. \\
\hline
\end{tabular}

Table II. LIST OF SYMBOLS

At any time instant $t$, let us define $p_{i}$ as the number of packets buffered by RCST $i$. $p_{i}$ is smaller or equal to the RCST $i$ 's buffer size. In this paper we assume that all RCSTs have the same buffer size $\beta$.

In any given time interval, there is a number of packets from RCSTs suffering $k$ or less levels of rain fading, which are needed to be fitted into MF-TDMA super frame.

A super frame consists of $N$ carriers. Each carrier has a fixed number of time slots, but possibly different bandwidth. In our framework as explained in Section 2, all carriers have the same number of time slots S. The authors in [8] refer to them as cells. A time slot can only be used for one packet. The bandwidth required for time slot $i$ in carrier $n, w_{i, n}$, is determined by the NCC based on the atmospheric condition of the RCST. A carrier $n$ bandwidth is allocated as the largest bandwidth required for any time slot assigned to carrier $n: \max _{s=1 . . S} w_{i, s}$.

All Super frames consist of the same number of Frames $L$. The objective of a time slot assignment method is to minimize the used portion of the MF-TDMA space to satisfy RCSTs requests. 


$$
\text { minimize } \sum_{n=1}^{N} \max _{s=1 . . S} w_{n, s}
$$

The current model describes one type of traffic, namely, CRA with guaranteed services. In the guaranteed services RCSTs are always allocated the requested rate, which means the Super Frame includes all the packets. This constraint is shown in Equation 8. The constraint in Equation 9 makes sure that the total number of packets generated by RCST $i$ doesn't exceed the available buffer size.

$$
\begin{aligned}
& \text { subject to } \sum_{i=1}^{u} p_{i} \leq N \cdot S \\
& p_{i}<=R_{i} \cdot \frac{T \cdot S}{\text { Bits per slot }}, \forall i
\end{aligned}
$$

Finally, the constraint in Equation 9 ensures meeting the targeted data rate for all RCSTs.

\subsection{The Proposed algorithm overview}

Any Media Access Control (MAC) algorithm breaks into stages starting with SNR measurement or prediction algorithms explained in Section 3. BoD calculation is the next stage in a MAC algorithm, highlighted in Section 2. BoD control calculates the actual portion required to satisfy an RCST request. Finally, the last stage in the MAC is the radio resource and time slots allocation. Radio Resource Allocation (RRM) controls bandwidth sharing among RCSTs conditioned by constraints such as priority, fairness, and QoS policy. The radio resource and time-slot allocation algorithms proposed in $[5,6]$ aim to maximize the network throughput. Maximizing network throughput gives RCSTs with clear sky conditions the highest priority to be allocated because they demand less MFTDMA space. Using less MF-TDMA space maximizes the network throughput as more RCSTs are allocated to the given MF-TDMA space. In the case of different rain fading levels, in other words, rain granularity, the allocation priority is inversely proportional to rain fading level.

The proposed algorithm adopts the principle given in [5,6] for allocation priority, but it uses a different frame structure. The algorithm serves worse faded links first, then the links with less fading level, and so on so forth. In other words, the allocation algorithm serves RCSTs with level $\mathrm{k}$ fading then $k-1, k-2, \ldots, 1$, when there are $k$ groups of packets suffers level $k$ rain fading. The proposed algorithm tends to maximize the MF-TDMA space utilization shown in the objective function in Equation 7.

In this paper, Constant Resource Allocation (CRA) is being used as a traffic class. Using CRA traffic class is a valid approach to study dynamics of rain fading in resource allocation schemes in satellite networks for simplification purposes $[5,6]$. In other words, any change in resource allocation is purely governed by rain fading.

The proposed MAC algorithm consists of two steps inspired by[6]:

1. Resource Calculation Algorithm (RCST side), see Figure 6: the first step is the SNR calculation, either by estimation using the model in [6], or measuring link actual rain fading level in real time, if there is an accessible meteorological database available. This task can be done by NCC or each RCST individually. All RCSTs, then, transmit a request identifying the targeted data rate $R_{i}$ and rain fading level $r_{i}$ to the NCC.

2. Radio Recourse Allocation (NCC side), see Figure 7: NCC collects all requests $R_{i}$ and the instantaneous rain fading levels information $r_{i}$ for all RCSTs. From $R_{i}$ and $r_{i}$ the NCC calculates the required bandwidth to satisfy each request. The next step, NCC generates Super Frame structure by running the RRA and time slot allocation algorithm. Finally, from Super Frame, the NCC generates the TBTP and transmits it to all RCSTs.

In our framework, we impose constraints on the frame structure extracted from ETSI in [14], as explained in Section 2.2. Frames are assumed to have a fixed duration, with a dynamic structure. A super frame is composed of a fixed number of sequential frames in a raw. 
Resource calculation algorithm (RCST $i$ side)

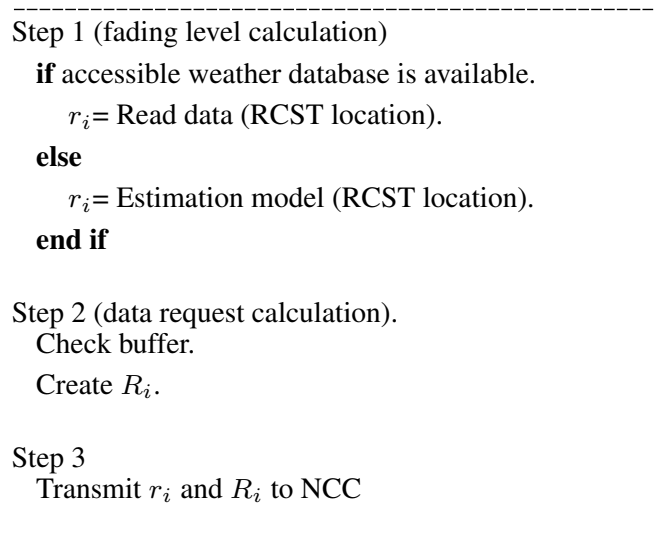

Figure 6. Allocation algorithm RCST $i$ side

The main idea behind the proposed time slots allocation algorithm is to bundle together packets coming from RCSTs with the same rain fading level (the same ACM mode).

\section{Radio Recourse Allocation (NCC side)}

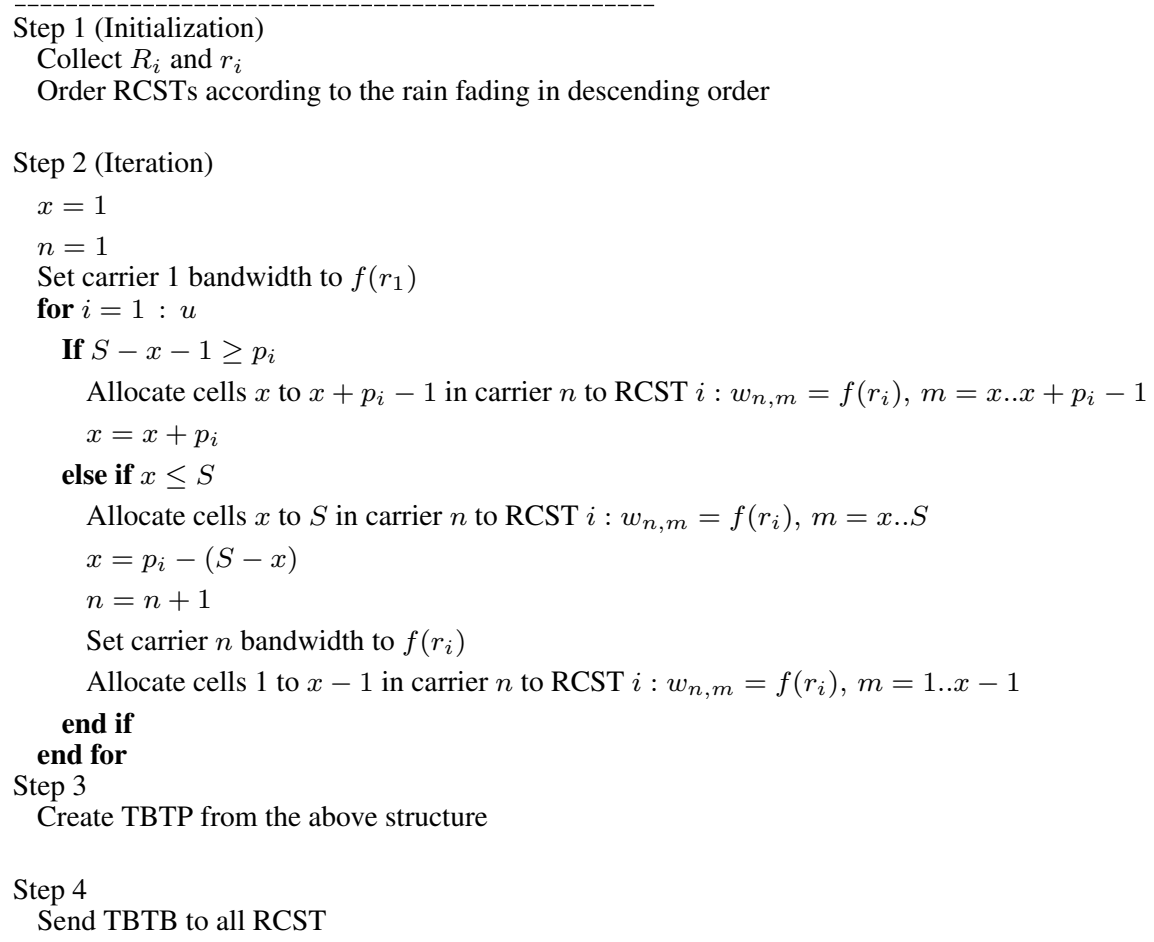

Figure 7. Allocation algorithm NCC side

The algorithm is working under the assumption that the used bandwidth does not exceed the total frame bandwidth, where $B^{j}$ is defined as the bandwidth assigned by the BoD to mitigate level $j$ rain effects. Scheduling packets from RCSTs with the same rain fading level into carriers of the same bandwidth reduces the amount of unused space in each MF-TDMA frame.

There may still be a situation when a portion of a carrier in a frame is unused because there are not enough packets at the given rain fading level to fill in all the time slots in that carrier. In the 
proposed algorithm, we introduce a time window to further improve carrier utilization. The time window is defined as the time interval required to transmit $L$ MF-TDMA frames. Within this time period, requests will be stored in RCSTs buffers to be transmitted in the next time window. The algorithm looks at requests after each window. Therefore, instead of generating a Terminal Burst Time Plan (TBTP) for each super frame, there will be one TBTP generated for each time window. This is illustrated in Figure 8.

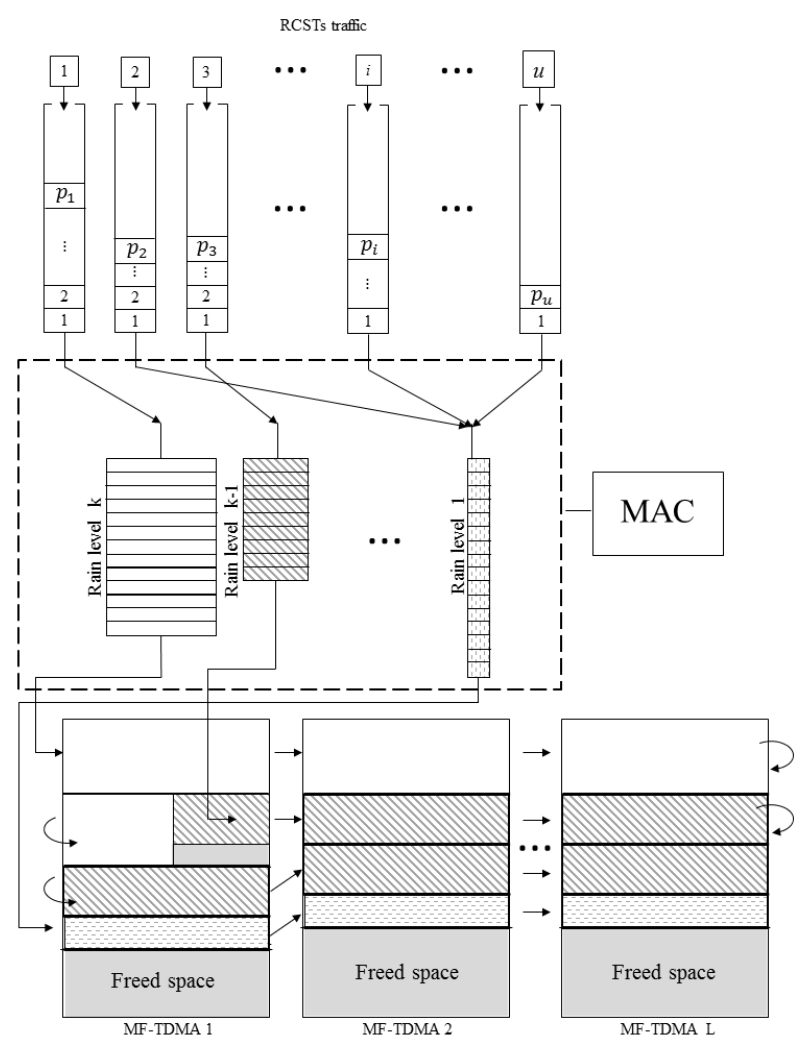

Figure 8. MAC serving $K$ packet groups into $L$ frames

Having the time window helps capturing more packets at a given rain fading level, which can then be transmitted within a carrier of an appropriate bandwidth. This means that less frames may be required to transmit them. The freed up frames in a given time window can be used to carry traffic from users with best effort level of services. For example, instead of transmitting one packet per carrier in 10 frames, the 10 packets can be buffered and transmitted in the next time window within a single frame. That means we will not need to reserve bandwidth for this rain fading level in the other 9 frames, and freed up bandwidth will be available for other services. This improvement in bandwidth utilization comes at the price of an added delay, which can be given in the following formula:

$$
\text { delay bound }=L \cdot \text { frame duration }+ \text { propagation delay }
$$

A downside of using this algorithm is that it will delay and queue data even for unfaded satellite links. One possible way to address it is to use a dynamic window size dependent on the rain fading conditions as well as burstiness of traffic. The algorithm can be illustrated graphically using Figure 10, which shows how traffic from different RCSTs is grouped according to their rain fading levels. Each group of packets then is sequentially scheduled by MAC into the corresponding carriers across the MF-TDMA frames in the time window. 


\subsection{Theoretical Analysis}

Our goal is to find an assignment of packets generated by RCSTs with different levels of rain to cells within a super-frame to minimize the total bandwidth required for the super-frame.

For a given set of packets, an assignment of packets to cells in the super-frame can be characterized by a bandwidth matrix $W=\left(w_{n, s} \mid n=1 . . N, s=1 . . S\right)$ where $N$ is the number of carriers in the super-frame, $S$ is the number of time slots, and $w_{n, s}$ is the bandwidth required to transmit the packet assigned to cell in carrier $n$, time slot s according to the rain level of the RCST, which generated that packet. If no packet is assigned to a cell, then $w_{n, s}=0$. The bandwidth of a given carrier is determined by the maximum bandwidth required to transfer any packet assigned to that carrier.

Without loss of generality, we can assume that

$$
\forall i, j: i<j \Longrightarrow \max _{s=1 . . S} w_{i, s} \geq \max _{s=1 . . S} w_{j, s}
$$

that is, we can assume that carriers are ordered by their bandwidth. This ordering does not have to correspond to the order of carrier frequencies. In the actual MF-TDMA frame structure, a carrier can be assigned to any frequency.

The total bandwidth of a super-frame can be calculated as:

$$
\sum_{n=1}^{N} \max _{s=1 . . S} w_{n, s}
$$

Lemma 1:

For any assignment, where $w_{n, s}=0$ for some $\mathrm{s}$ and $n<N$, there is an assignment with at least the same or smaller total bandwidth, where $w_{n, s}>0$ for the same $n$ and $s$.

Proof:

1. It follows from our carriers ordering assumption, that

$$
\forall i, j: i<j \Longrightarrow \max _{s=1 . . S} w_{i, s} \geq \max _{s=1 . . S} w_{j, k}
$$

for any $k=1 . . S$. Let $w_{n, s}=0$ for some $n<N$ and $s$. Then we can reassign any packet transmitted on any carrier $n^{*}>n$, to carrier $\mathrm{n}$, time slot $\mathrm{s}$ without increasing the bandwidth required for carrier $\mathrm{n}$ and, hence, without increasing the total bandwidth required.

2. It is possible that by repeated reassignment, we may reassign all packets from carrier $N$. As per point 1 , this will not change the bandwidth required for carriers $1 . . N-1$. However, we will be able to reduce the total bandwidth required, as carrier $N$ will not be needed after reassignments.

Lemma 1 allows us to consider only assignments where $w_{n, s}>0$ for all $s$ and $n<N$ to minimize the total bandwidth required. Therefore, in the subsequent analysis we assume that $w_{n, s}>0$ for all $s$ and $n<N$, that is there are no empty cells in carriers other than $N$.

Definition 1:

Let us define an assignment as ordered if

$$
\forall i, j: i<j \Longrightarrow \min _{s=1 . . S} w_{i, s} \geq \max _{s=1 . . S} w_{j, s}
$$

\section{Lemma 2:}

For any given set of packets, any two ordered assignments of those packets would require the same total bandwidth.

Proof: 
1. By definition of the total bandwidth, if two assignments are characterized by the same bandwidth matrix $W=\left(w_{n, s} \mid n=1 . . N, s=1 . . S\right)$, then they will require the same total bandwidth.

2. Let there be two ordered assignments characterized by two different bandwidth matrices $W$ and $W^{*}$. Both matrices will have the same number of columns $S$, because the number of time slots in a super-frame is fixed. Since both assignments are for the same number of packets, under our assumption of no empty cells other than in the last carrier, both matrices will have the same number of rows $N$.

3. Let us assume that the total bandwidth required for $W$ is different from the total bandwidth required for $W^{*}$.

4. Under the assumption of ordered carriers

$$
\max _{s=1 . . S} w_{1, s}=\max _{s=1 . . S} w_{1, s}^{*}
$$

5. Without loss of generality, let there be carrier $i>1$, such that

$$
\max _{s=1 . . S} w_{i, s}<\max _{s=1 . . S} w_{i, s}^{*}
$$

6. Since both assignments are ordered,

$$
\min _{s=1 . . S} w_{i-1, s} \geq \max _{s=1 . . S} w_{i, s}
$$

and

$$
\min _{s=1 . . S} w_{i-1, s}^{*} \geq \max _{s=1 . . S} w_{i, s}^{*}
$$

Also, the bandwidth required to transmit any packet assigned to any carrier greater or equal to $i$ in $W$ will be less or equal to $\max _{s=1 . . S} w_{i, s}$.

7. Therefore, for the condition in point 5 to hold, there must be a packet that is assigned to a carrier less than $i$ in $W$, but that is assigned to carrier $i$ in $W^{*}$.

8. Since assignment $W$ is ordered and from point 7

$$
\min _{s=1 . . S} w_{i-1, s} \leq \max _{s=1 . . S} w_{i, s}^{*}
$$

9. Under our assumption of no empty cells other than in the last carrier and from point 7 , there must be a packet that is assigned to a carrier greater or equal to $i$ in $W$, but that is assigned to a carrier less than $i$ in $W^{*}$.

10. Since assignment $W^{*}$ is ordered and from point 9

$$
\max _{s=1 . . S} w_{1, s}=\max _{s=1 . . S} w_{1, s}^{*}
$$

11. Putting together equations in points 6,8 , and 10

$$
\begin{aligned}
& \min _{s=1 . . S} w_{i-1, s} \leq \max _{s=1 . . S} w_{i, s}^{*} \leq \min _{s=1 . . S} w_{i-1, s}^{*} \\
& \min _{s=1 . . S} w_{i-1, s}^{*} \leq \max _{s=1 . . S} w_{i, s} \leq \min _{s=1 . . S} w_{i-1, s}
\end{aligned}
$$

It follows from the above equations, that the only possibility is that $\max _{s=1 . . S} w_{i, s}=\max _{s=1 . . S} w_{i, s}^{*}$, which is a contradiction with point 5 .

12. Therefore, $\max _{s=1 . . S} w_{i, s}=\max _{s=1 . . S} w_{i, s}^{*}$ for any $i>1$. This means that the total bandwidth required for both assignments is the same.

\section{Theorem 1:}

For a given set of packets, any ordered assignment of those packets minimizes the total bandwidth required.

Proof: 
1. Let there be an assignment of packets characterized by bandwidth matrix $W^{*}$ that minimizes the total required bandwidth.

2. Let us assume that this assignment is not ordered. Then there must exist a pair of carriers $i$ and $j, i<j$, such that

$$
\min _{s=1 . . S} w_{1, s}^{*} \leq \max _{s=1 . . S} w_{j, s}^{*}
$$

3. The bandwidth of the packet requiring the largest bandwidth in carrier $j$ is less then the bandwidth of the packet requiring the largest bandwidth in carrier $i$ (due to ordered carriers). Therefore, if we swap the packet requiring the largest bandwidth in carrier $\mathrm{j}$ with the packet requiring the smallest bandwidth in carrier $i$, then it will not increase the bandwidth of carriers $i$ and $j$. Therefore, the swap will not increase the total bandwidth required.

4. We can repeat the swapping in point 3 until there are no carriers left for which the condition in point 2 holds.

5. The new assignment in point 4 will be ordered and according to point 3 will have no greater total bandwidth than the initial assignment $W^{*}$.

6. According to Lemma 2, any ordered assignment for the given set of packets will have the same total bandwidth required. Therefore, any ordered assignment for the given set of packets will have no greater total bandwidth than assignment $W^{*}$.

As explained in Section 5.2, the proposed algorithm orders RCSTs by rain fading level, then fits their packets sequentially into the MF-TDMA space. Thus, the algorithm generates an ordered packet assignment and, hence according to Theorem 1, minimizes the total MF-TDMA bandwidth required.

\subsection{Simulation and Results}

To evaluate the performance of the proposed algorithm, MATLAB based simulations were used. The simulation uses the same RCST distribution technique described in Section 4. The data rate used is $144 \mathrm{~Kb} / \mathrm{s}$ (topology A) guaranteed throughput of a low rate video streaming traffic type, with burstiness of 4, 8, 16 and 32 with on/off sources. In the simulation, we assume the burstiness is measured over a single time window. Precisely, we assume that each RCST transmits at peak rate only for $1 / 4$ th , 1/8th, 1/16th and 1/32 of the time window duration. Each time slot has a capacity of $16 \mathrm{~Kb} / \mathrm{s}$ guaranteed throughput. Therefore, 10 time slots per time window must be allocated to each RCST to satisfy the targeted rate. The above numbers were obtained from calculation carried in $[5,10,15]$. In this version of the simulator, we considered one data type "video streaming" over one MF-TDMA of $20 \mathrm{MHz}$ bandwidth, with transmission frequency of $30 \mathrm{GHz}$.

\begin{tabular}{|c|c|c|c|c|c|}
\hline \multirow{2}{*}{$\begin{array}{c}\text { Peak } \\
\text { information } \\
\text { data rate }\end{array}$} & Slots per & carries & and per & frame & Carriers \\
\hline & $\begin{array}{c}\text { traffic } \\
\text { slots }\end{array}$ & $\begin{array}{l}\text { CSC/ } \\
\text { slots }\end{array}$ & $\begin{array}{c}\text { SYNC/ } \\
\text { slots }\end{array}$ & $\begin{array}{l}\text { Total } \\
\text { slots }\end{array}$ & $\begin{array}{l}\text { per } \\
\text { frame }\end{array}$ \\
\hline $144 \mathrm{~Kb} / \mathrm{s}$ & 9 & 1 & 2 & 10 & 60 \\
\hline $384 \mathrm{~Kb} / \mathrm{s}$ & 24 & 2 & 4 & 26 & 23 \\
\hline $1024 \mathrm{~Kb} / \mathrm{s}$ & 64 & 4 & 8 & 68 & 9 \\
\hline $2048 \mathrm{~Kb} / \mathrm{s}$ & 128 & 8 & 16 & 136 & 4 \\
\hline
\end{tabular}

Table III. FEATURES OF THE MF-TDMA RETURN LINK [15]

In the given simulation, 480 RCSTs were used with the same geographical distribution pattern described in Section 4.

The algorithm uses version 1 DVB-RCS system with 9 ACM FMT modes available, i.e., $k=9$ in this case. The simulation uses historical rain data, with a simulation duration of 1 month (February 2011 and 2012). 


\subsection{Numerical Results}

Figure 9 shows the amount of used bandwidth by the proposed algorithm and compares is to the base line described in the beginning of Section 5, which is the constant bandwidth allocation for the given distribution of RCSTs. We performed our experiments for traffic burstiness of 4, 8, 16, and 32. The overall bandwidth usage statistics can be represented as a Cumulative Density Function $(\mathrm{CDF})$ of the total bandwidth used. This is shown in Figure 10.
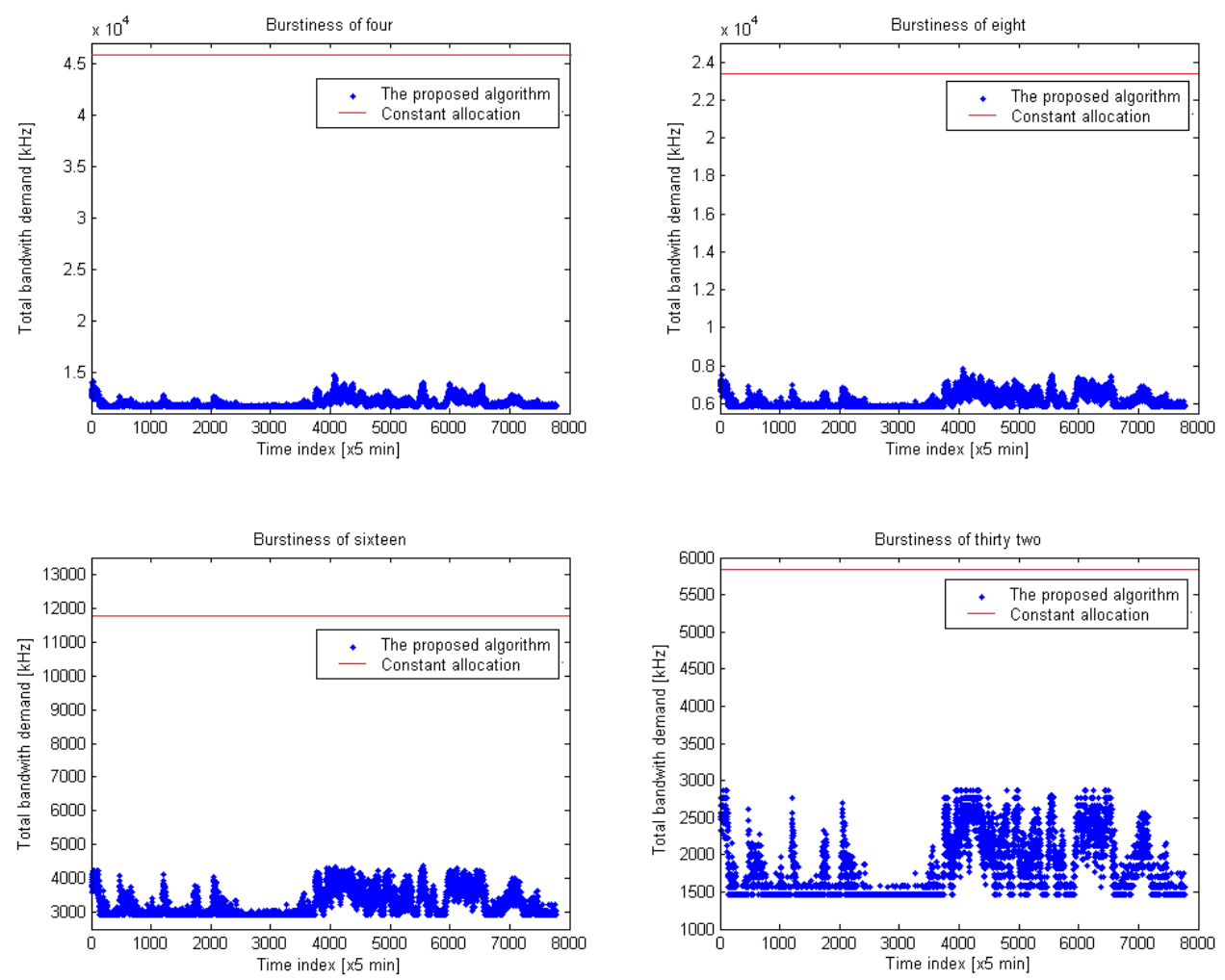

Figure 9. Total bandwidth demand

As it can be seen from the results shown in Figure 9 and 10, at any given time, 14.56, 7.8, 4.38, $2.89 \mathrm{MHz}$ or less of the bandwidth for the burstiness of $4,8,16,32$ respectively.

\section{CONCLUSION}

Rain attenuation plays important role in satellite communication due to the limited spectrum available. This paper presented a study of bandwidth utilization. We investigated the problem by looking at three aspects: rain granularity, RCSTs geographical locations, and radio resource allocation and time slot allocation.

We compared the effects of considering rain granularity with worst case scenario suggested by authors in $[7,10,14]$. We developed a simulation scenario using MATLAB based on real rain data for the UK and we used the ACM with constant bit rate as FMT. We measured the used bandwidth in the case of considering rain granularity, worse case scenario and fixed allocation for a duration of three months. Using rain granularity, i.e., dynamically changing the modulation scheme, can save up to $33 \%$ in compared with worse case scenario and $64 \%$ compared with fixed bandwidth allocation. 

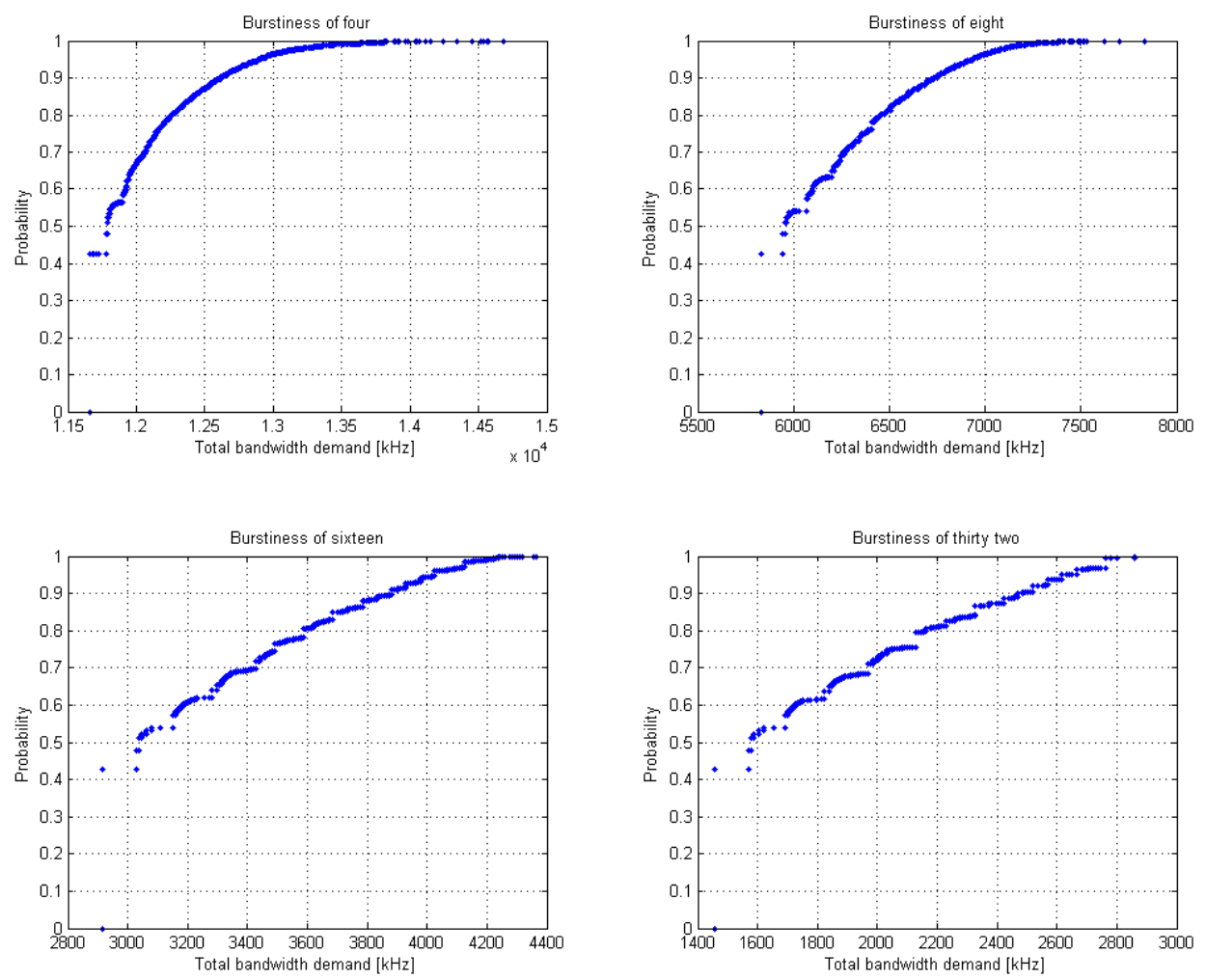

Figure 10. Total bandwidth demand CDF

Unlike other referenced approaches, where RCSTs are divided into only two groups of rainy and non-rainy terminals, the proposed algorithm bundles traffic requests into multiple groups corresponding to their individual rain fading levels. The algorithm also uses a time window to determine the expected delay bound, which consists of multiple consecutive MF-TDMA frames, to improve time slot utilization within each carrier and free up frame bandwidth for other services. The paper provided theoretical analysis of the time slot allocation problem and show that the proposed algorithm achieves theoretically optimal assignment. The proposed algorithm is evaluated using MATLAB simulation with real historical rain data. The proposed time slot allocation algorithm showed a significant improvement in utilisation: only $35.2 \%$ to $40 \%$ of the available frame bandwidth was used at any given time in comparison with the constant bandwidth allocation.

In our future work, we will study the effects of RCST geographical distribution and using rain statistical information on call admission control process. The current version of the algorithm will also be expanded to include different types of services, which means using different radio resource sharing policy rather the round robin in the case of guaranteed services.

\section{REFERENCES}

1. ETSI EN 301790 (V1.3.1) Digital Video Broadcasting (DVB); Interaction Channel for Satellite Distribution Systems, Final Draft, 2002-11.

2. ETSI EN 301790 (V1.5.1) Digital Video Broadcasting (DVB); Interaction Channel for Satellite Distribution Systems, Final Draft, 2009-05.

3. Chao HJ, Guo X. Quality of service control in high-speed networks. John Wiley: New York, 2002.

4. Y Feng. Resource allocation in Ka-band satellite systems. Dept. of Elecrical and Computer Engineering, University of Maryland, 2001.

5. E Noussi. A study of fade mitigation and resource management of satellite networks in rain condition. Dept. of Eelectroic and Computer Enginnering, University of Portsmouth, 2008.

6. Petraki DK, Anastasopoulos MP, Cottis PG. Dynamic resource allocation for DVB-RCS networks. International Journal of Satellite Communications and Networking 2008; 26:189-210. 
7. Pace P, Aloi G, Marano S. Efficient real-time multimedia connections handling over DVB-RCS satellite system. proceedings of the GLOBECOM '04, Texas, USA, vol. 5, no., pp. 2722- 2727, Nov. 2004.

8. Al-Mosawi M, Khusainov R, Gremont B. A Real Time Algorithm for Bandwidth and Time-slot Assignment for Rain Faded DVB-RCS Systems.proceedings of the Fourth International Conference on Advances in Satellite and Space Communications, in proc. of SPACOMM '12, Mont-Blanc, pp. 001-006, Apr. 2012.

9. Noussi E, Gremont B, Filip M. Integration of fade mitigation technique within centrally managed MF-TDMA DVB-RCS networks. International Journal of Space Communication DOI: 0924-8625/06.

10. Ki-Dong L, Kun-Nyeong C, A real-time algorithm for timeslot assignment in multirate return channels of interactive satellite multimedia networks. IEEE Journal on Selected Areas in Communications 2004 DOI: 10.1109/JSAC.2004.823433.

11. Guerin R, Ahmadi H, Naghshineh M. Equivalent capacity and its application to bandwidth allocation in high-speed networks. IEEE Journal on Selected Areas in Communications 1991 DOI:10.1109/49.103545.

12. Perros HG, Elsayed KM. Call admission control schemes: a review. IEEE Communications Magazine 1996 DOI:10.1109/35.544197.

13. Breslau L, Jamin S, Shenker S, Comments on the performance of measurement-based admission control algorithms. proceedings of the Nineteenth Annual Joint Conference of the IEEE Computer and Communications Societies, in proc. of INFOCOM 2000, Tel Aviv, vol.3, pp.1233-1242, Mar. 2000.

14. Morell A, Seco-Granados G, Vazquez-Castro MA. SAT03-3: Joint Time Slot Optimization and Fair Bandwidth Allocation for DVB-RCS Systems. proceedings of the Global Telecommunications Conference, in proc. of GLOBECOM '06, Taxas, pp.1-5, Nov. 2006.

15. Pace P, Aloi G, Marano S. Multimedia traffic admission schemes comparison for satellite systems. proceedings of Vehicular Technology Conference, in proc. of VTC2004, Milan, vol. 6, pp. $4022-4026$ Sep. 2004.

16. Jamin. A Measurement-based admission control algorithm for integrated services packet networks. Dept. of Computer Science, University of Southern California, 1996.

17. Genin D, Marbukh V. Bursty fluid approximation of TCP for modeling internet congestion at the flow level. proceedings of the 47th Annual Allerton Conference on Communication, Control, and Computing, in proc. of Allerton 2009, Allerton, vol. 30, pp.1300-1306, Sep. 2009 DOI: 10.1109/ALLERTON.2009.5394525.

18. T Kiong. Connection admission control in multi service networks: simplicity versus efficiency. Dept. of Electrical and Electronic Engineering, University of Melbourne, 2002.

19. De Rango F, Tropea M, Fazio P, Marano S. Call admission control with statistical multiplexing for aggregate MPEG traffic in a DVB-RCS satellite network. proceedings of the Global Telecommunications Conference, in proc. of GLOBECOM '05, Missouri, vol. 6, pp. 3232-3236, Dec. 2005 DOI: 10.1109/GLOCOM.2005.1578372.

\section{AUTHOR'S BIOGRAPHIES}

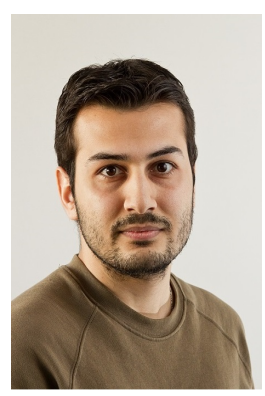

Mohamed Al-mosawi was born in Basra, Iraq, on April 1987. He received his Bachelor degree in Communication systems Engineering from University of Portsmouth, UK, in 2009, and M.Sc degree in Communication Networks Planning and Managements from the University of Portsmouth, UK, in 2010. He is currently working toward Dr. Eng degree in the same university. His major research area is in QoS management, call admission control, and Radio Resource Allocation for VSAT Network in presence of Rain. He served as a program committee member or reviewer for a number of international conferences and workshops. He is a member of Institute of Engineering.

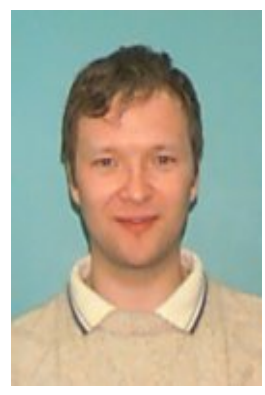

Rinat Khusainov received his Diploma in Computer Engineering from Chelyabinsk State Technical University, Russia, in 1997, and M.Sc and Ph.D degrees in Computer Science from University College Dublin, Ireland, in 1999 and 2004. He has extensive expertise in embedded and distributed systems, computer networks, information retrieval, artificial intelligence, and machine learning, with research and development experience working on projects funded by Irish and UK governments as well as the European Commission. Dr Khusainov has published over 40 refereed journal and conference papers and served as a program committee member or reviewer for a number of international conferences and workshops. He is a member of Institute of Engineering and Technology and a fellow of Higher Education Academy. 


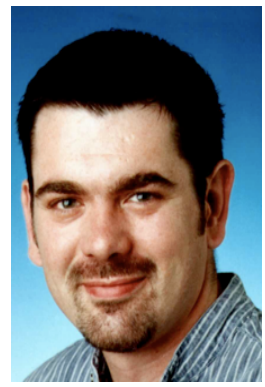

Boris Gremont received the DUT from the University Institute of Technology (IUT) of Grenoble, France, in 1992, and the Bachelor in Engineering (First Class Honors) in electronic engineering and Ph.D. degrees from Coventry University in 1994 and 1998, respectively. After two years of Postdoctoral research with the Microwave Telecommunication Systems Research Group (MTSRG), University of Portsmouth, he was appointed as a Senior Lecturer with the Department of Electrical and Electronic Engineering in 1999. He is teaching mathematics, communication systems, as well as data communications and networks at undergraduate and postgraduate levels. He was also involved in the European COST 280 action Propagation Impairment Mitigation for Millimeter Wave Radio Systems and also contributes to the U.K.s Fractal Rain Modeling for Millimeter Wave Propagation project (RAINMAP). 